\title{
MESSIER: exploring the ultra-low surface brightness universe with a curved focal plane based satellite
}

CNRS-LAM: Simona Lombardo*, E. Muslimov, E. Hugot, G. Lemaitre, M. Roulet, M. Ferrari CNRS-LERMA: D. Valls-Gabaud

\section{*simona.lombardo@lam.fr}

ICSO Conference, Chania, Greece, 11.10.2018

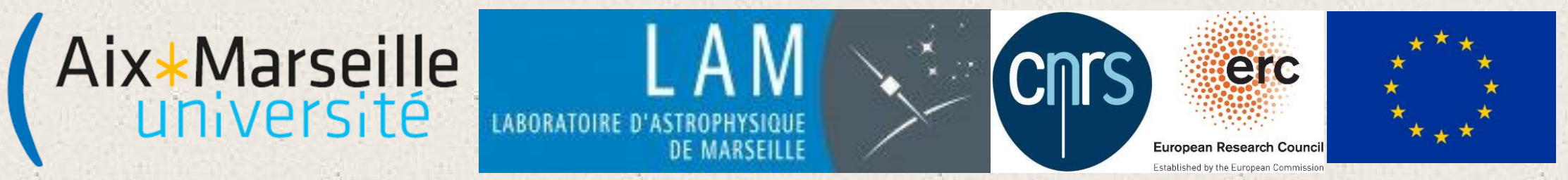




\section{Ultra-low surface brightness universe: Cosmic web}

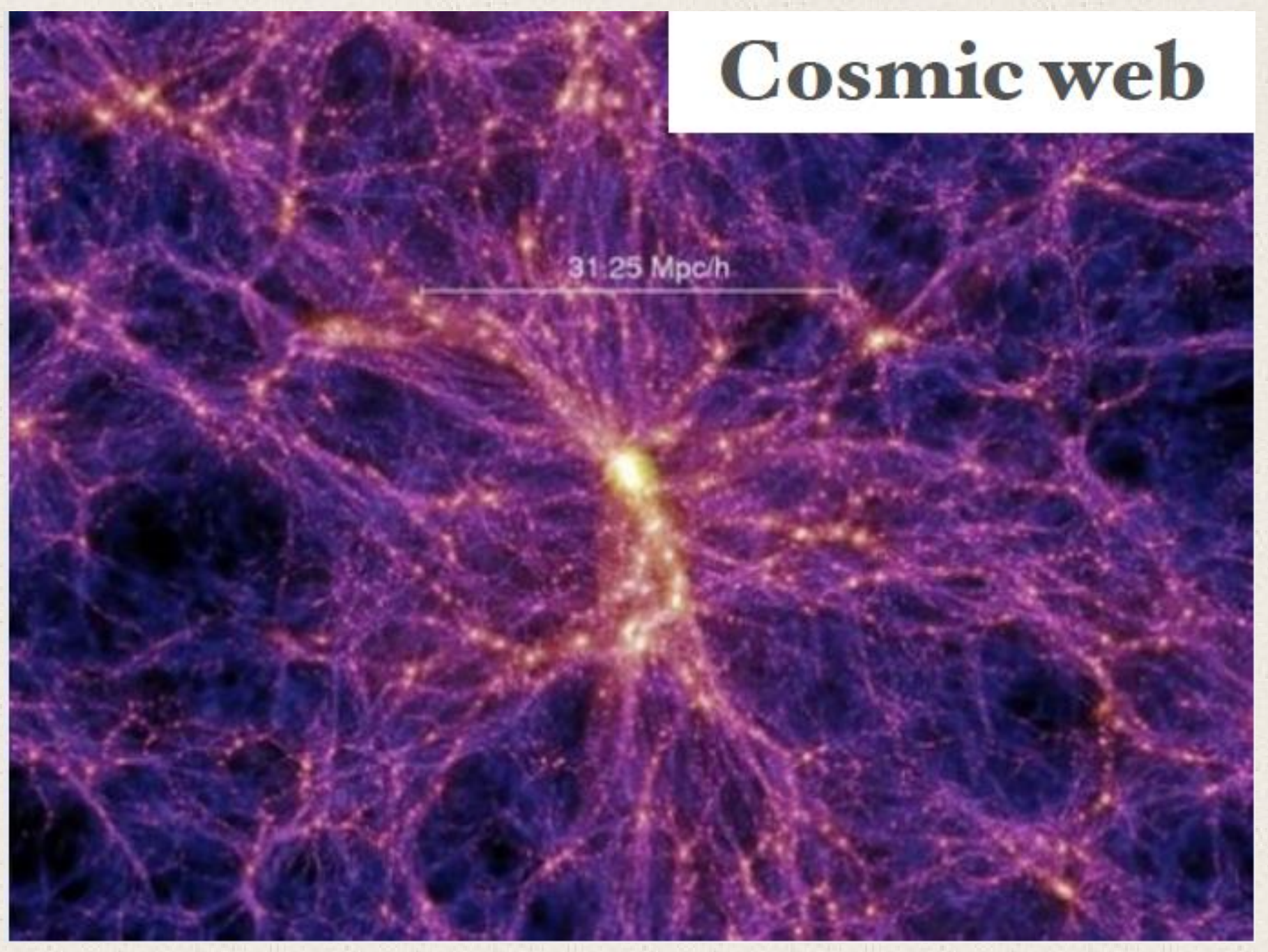

Springel, 2015

Brightness of 37

$\mathrm{mag} / \operatorname{arcsec}^{\wedge} 2$ in UV 


\section{Ultra-low surface brightness universe: NGC 5907}

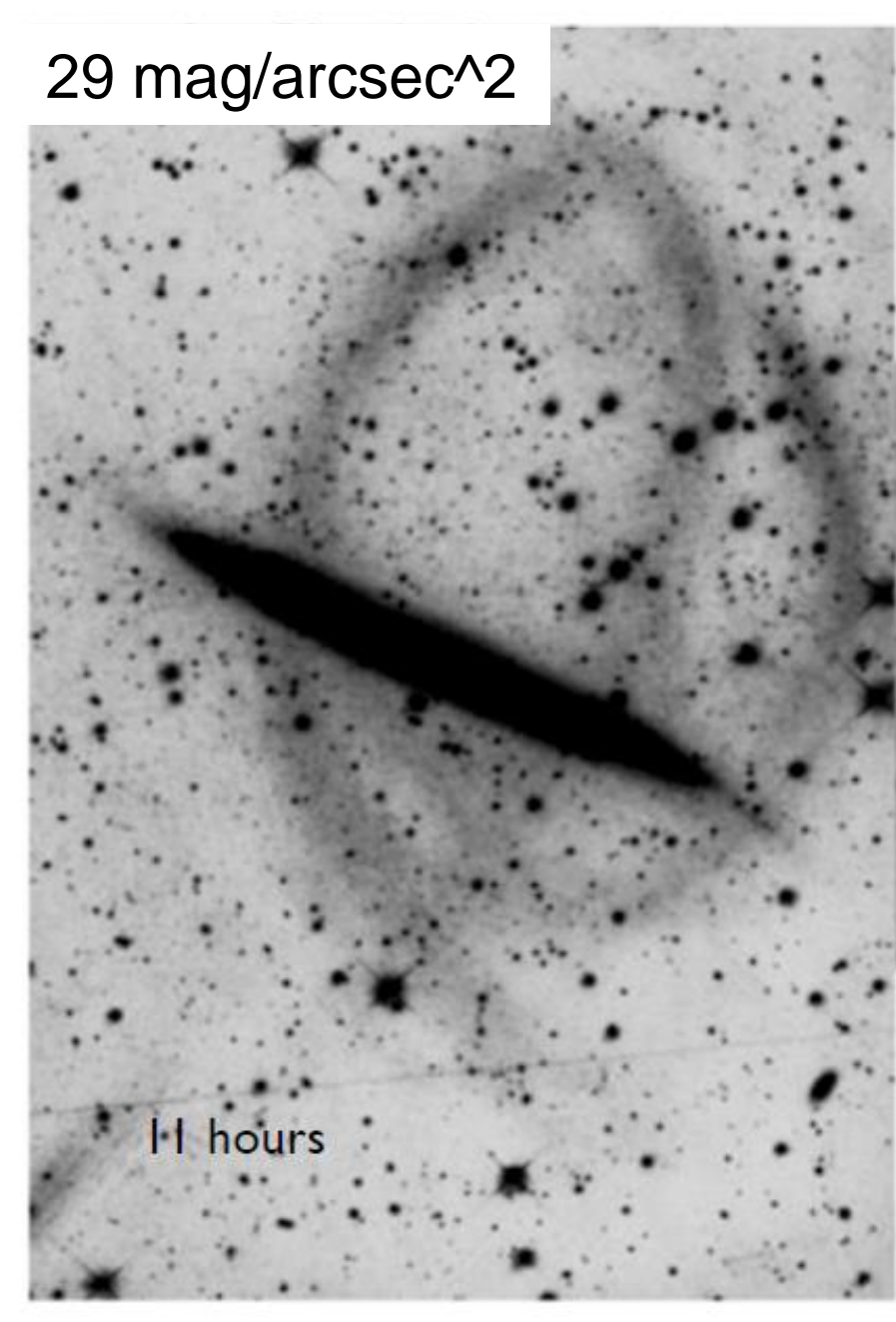

$0.5 \mathrm{~m} \mathrm{f} / 8 . \mathrm{I}$

Martinez Delgado et al. 2008

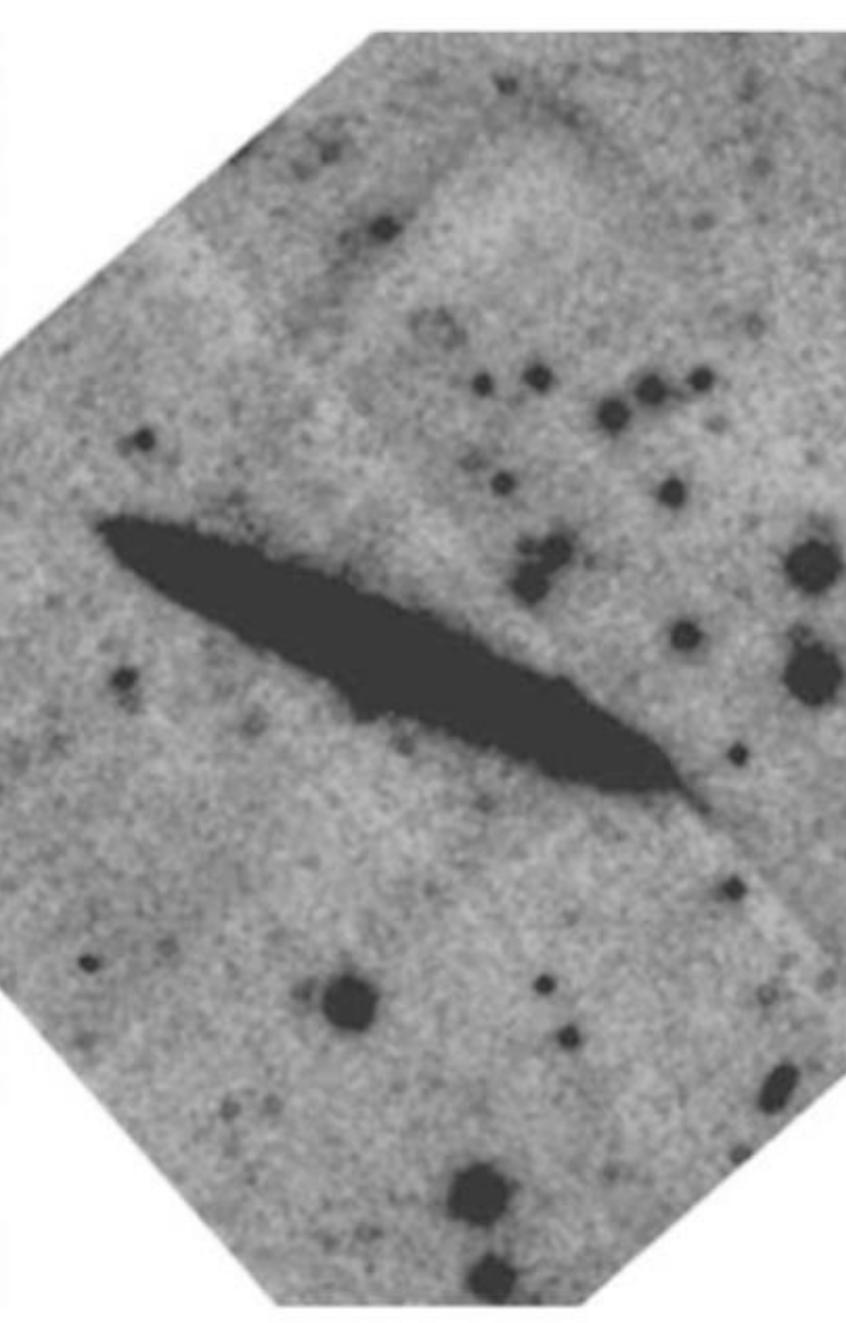

SDSS

Miskolczi et al. 2OII

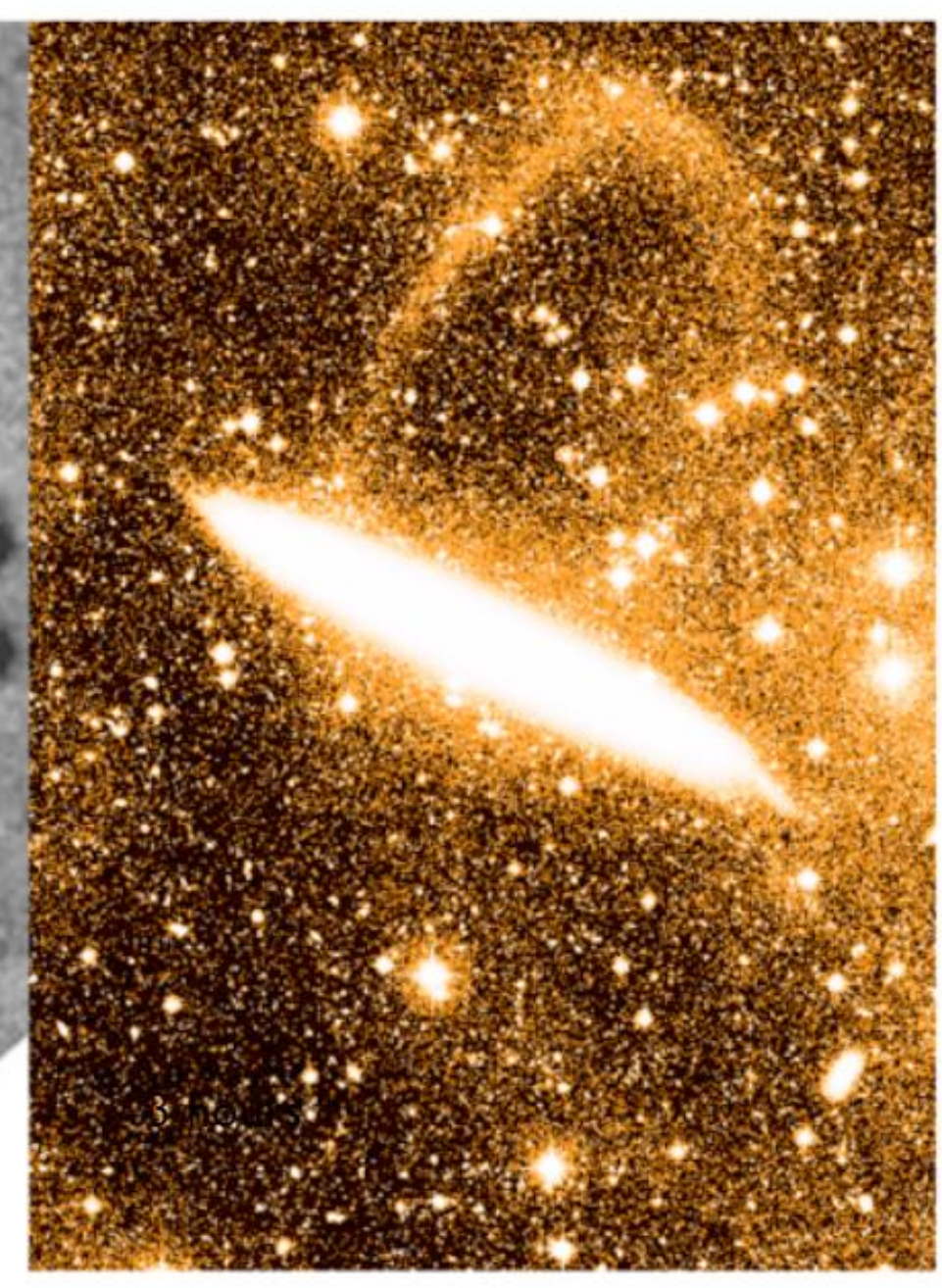

Credit Image: Valls-Gabaud
CFHT

Ibata et al. 2OII 


\section{Ultra-low surface brightness universe}

\section{REQUIREMENTS:}

- Observing from space (no molecular scattering,

lower and stable foreground)

- Low PSF wings (need to reach the faintest LSB levels)

- No refractive elements (no internal scattering, no Cerenkov) 


\section{Proposed fully reflective space-based MESSIER}

Quantity

Field of view

Central obscuration

Wavelength range

Diameter

Survey solid angle
Value

$2^{\circ} \times 4^{\circ}$

none

$150-1000 \mathrm{~nm}$

$50 \mathrm{~cm}$

$4 \pi$

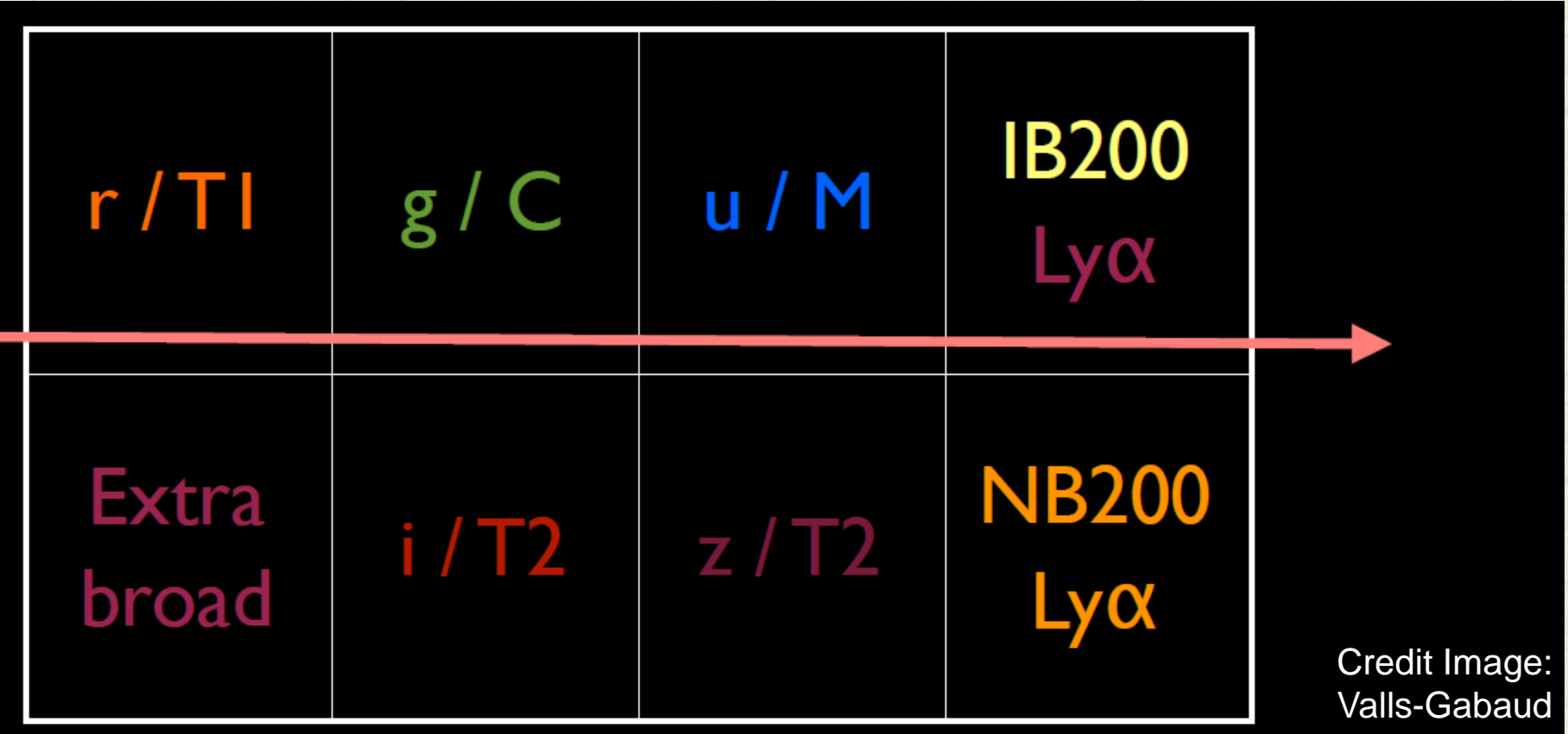

- 8 independent CCD for drift-scan mode

- QE of each CCD optimized for filter/band 


\section{Ground-based pathfinder}

Fully reflective Schmidt design to be installed in Tenerife/La Palma

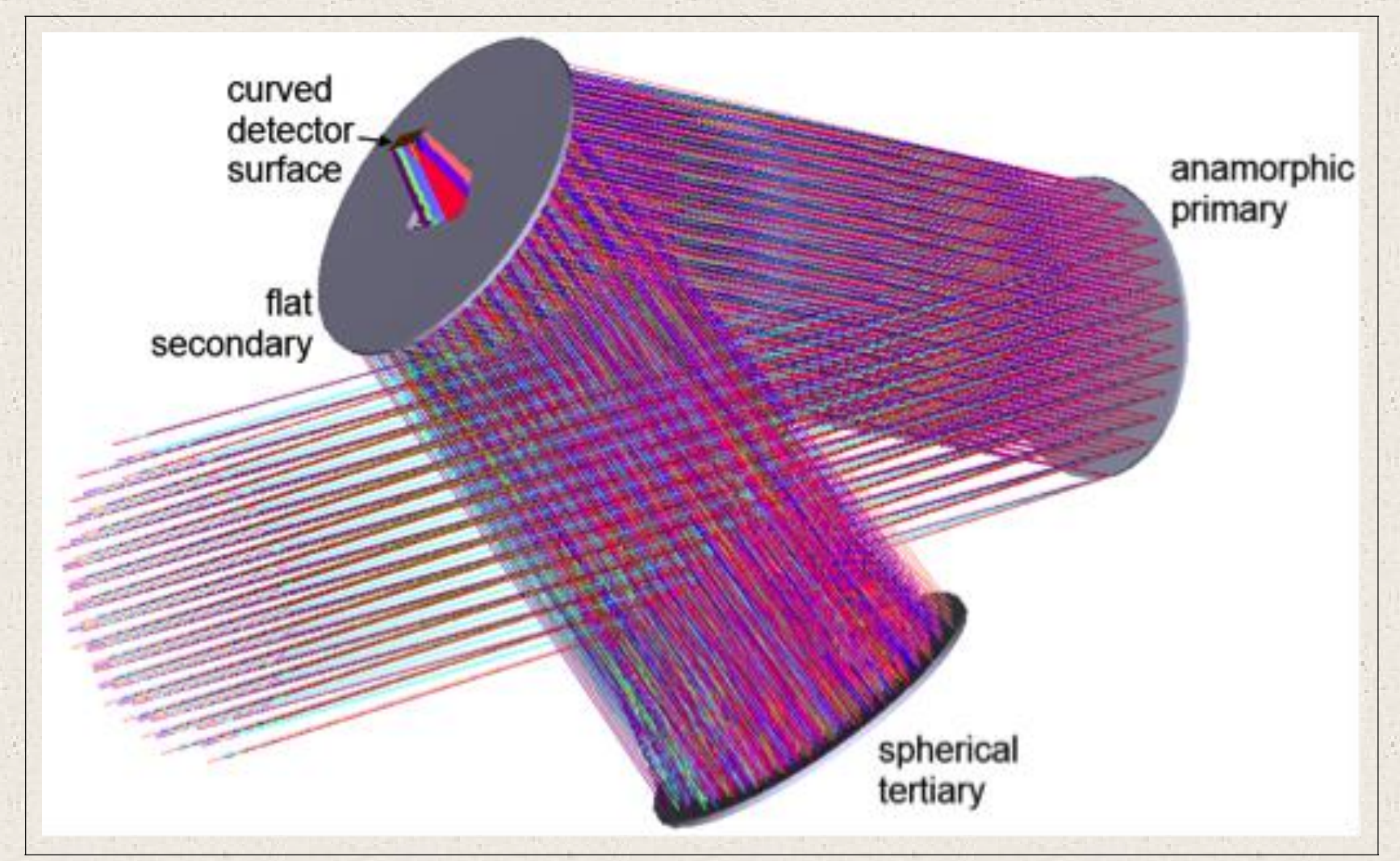

\begin{tabular}{lll}
\hline Parameter & Symbol & $\begin{array}{l}\text { Value } \\
\text { (pathfinder) }\end{array}$ \\
\hline $\begin{array}{l}\text { Field of } \\
\text { view }\end{array}$ & FoV & $1.6 \times 2.6$ \\
$f$-ratio & $\Omega$ & 2.5 \\
$\begin{array}{l}\text { Primary } \\
\text { diameter }\end{array}$ & M1 & $356 \mathrm{~mm}$ \\
$\begin{array}{l}\text { Distortion } \\
\text { Simot rms }\end{array}$ & $\rho$ & $<0.5 \%$ \\
Spotius & & $<12 \mu \mathrm{m}$ \\
\hline
\end{tabular}

Muslimov et al., 2017, Applied Optics, 56, 8639 


\section{State-of-the-art PSF calculations}

\section{Photon Monte CARLO SimUlations:}

- Photons propagation by raytracing

- Astrophysical source

- Atmospheric extinction

- Optical elements

- CCD effects 


\section{Photon Monte Carlo simulations}

Pathfinder with one necessary

refractive element:

g-band filter + cryostat window

Curved CCD detector 


\section{Simulated star at the center of the field}

1 pix $=2.32 "$

FoV $1.6^{\circ} \times 2.6^{\circ}$

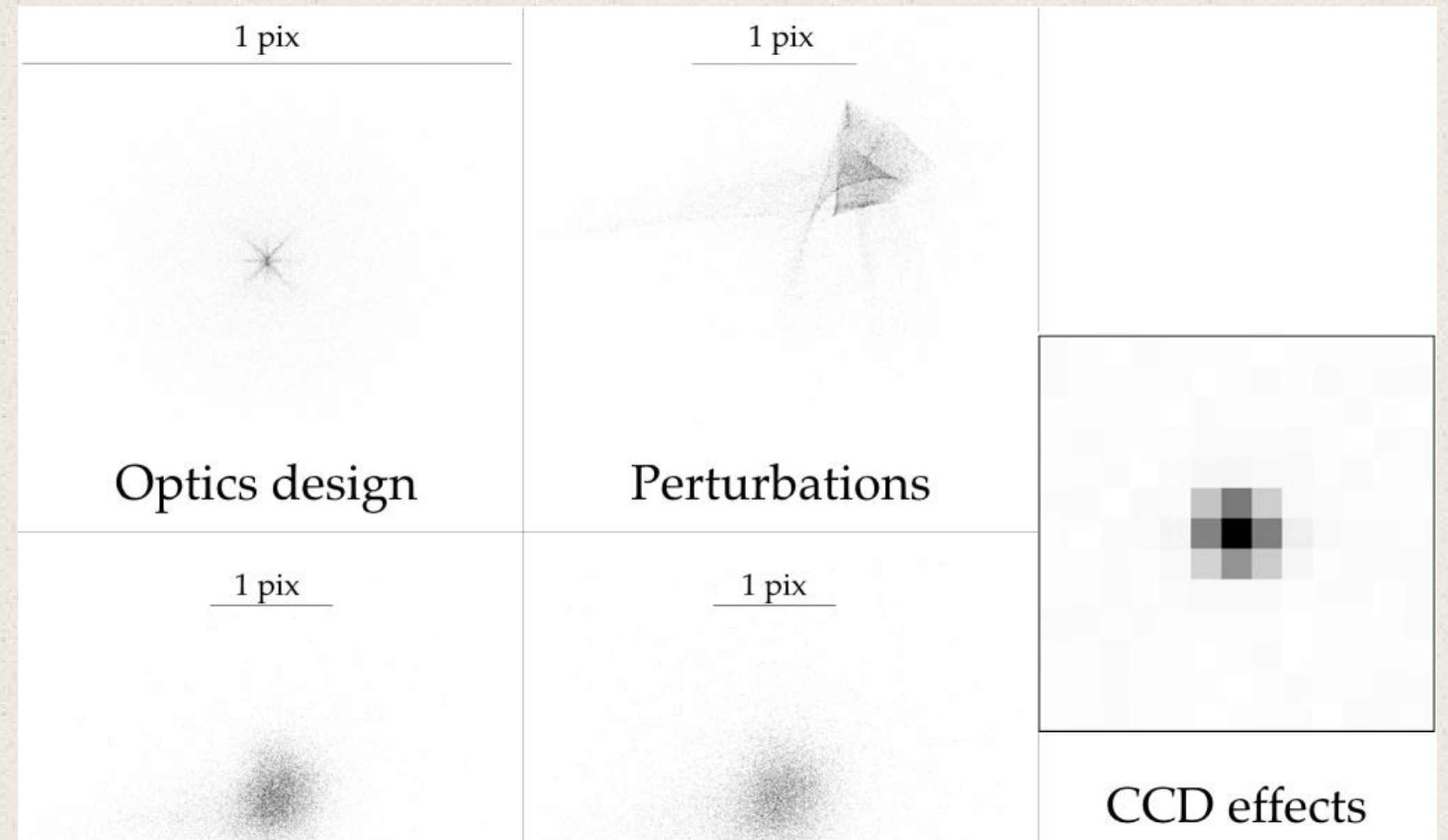




\section{Simulated star at the corner of the field}

1 pix $=2.32 "$

FoV $1.6^{\circ} \times 2.6^{\circ}$

The image in the focal plane is uniform in the full field!

\section{1 pix}

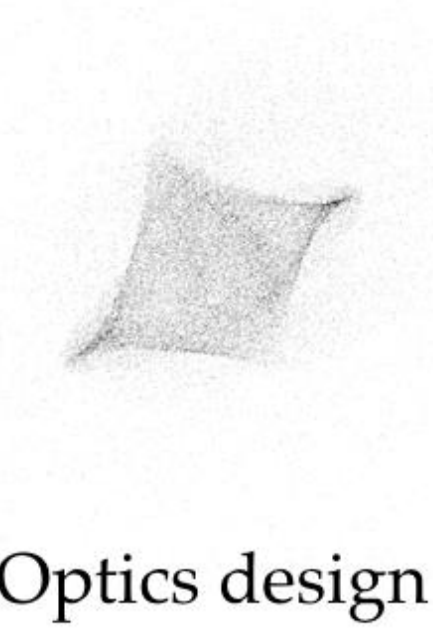

1 pix

1 pix 


\section{Single star simulation}

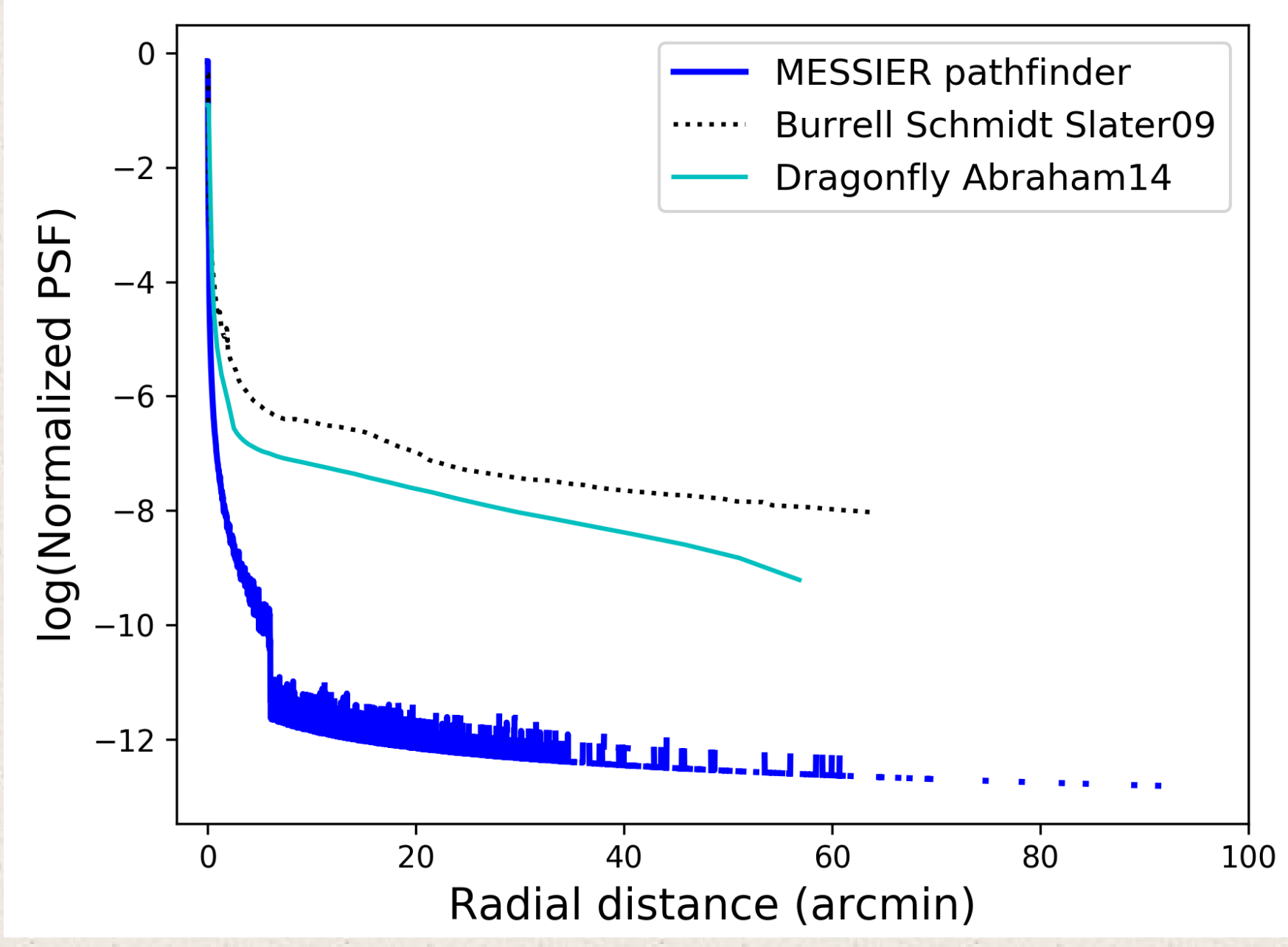

Data binned every 2.32" (1 pixel)

Star at the center of FoV

VERY LOW PSF WINGS IN

FULL FOV 


\section{Single star simulation}

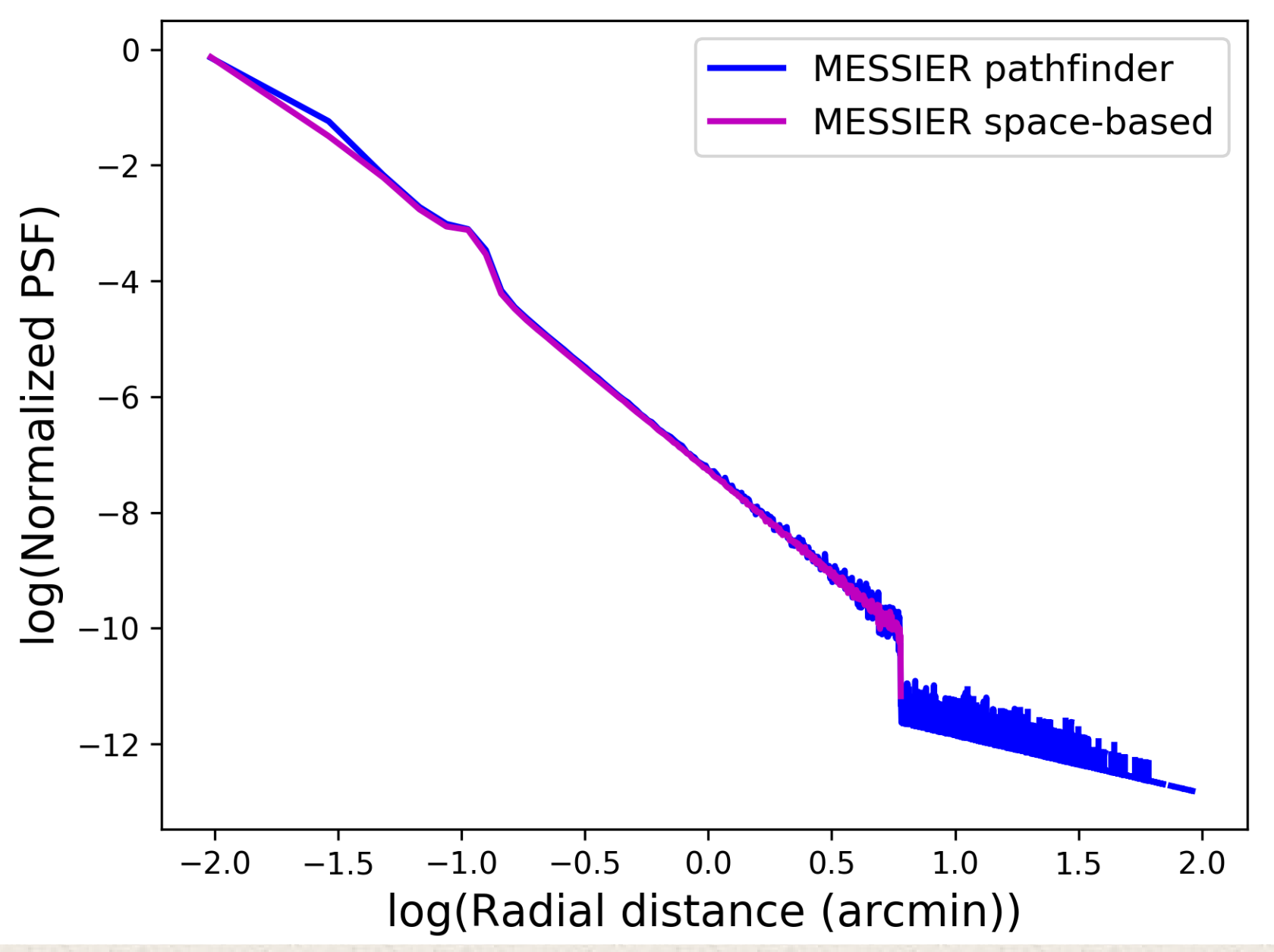

Data binned every 2.32" (1 pixel)

Star at the center of FoV

VERY LOW PSF WINGS IN

FULL FOV 


\section{U-LSB objects drift scan simulation $-5^{\prime}$}

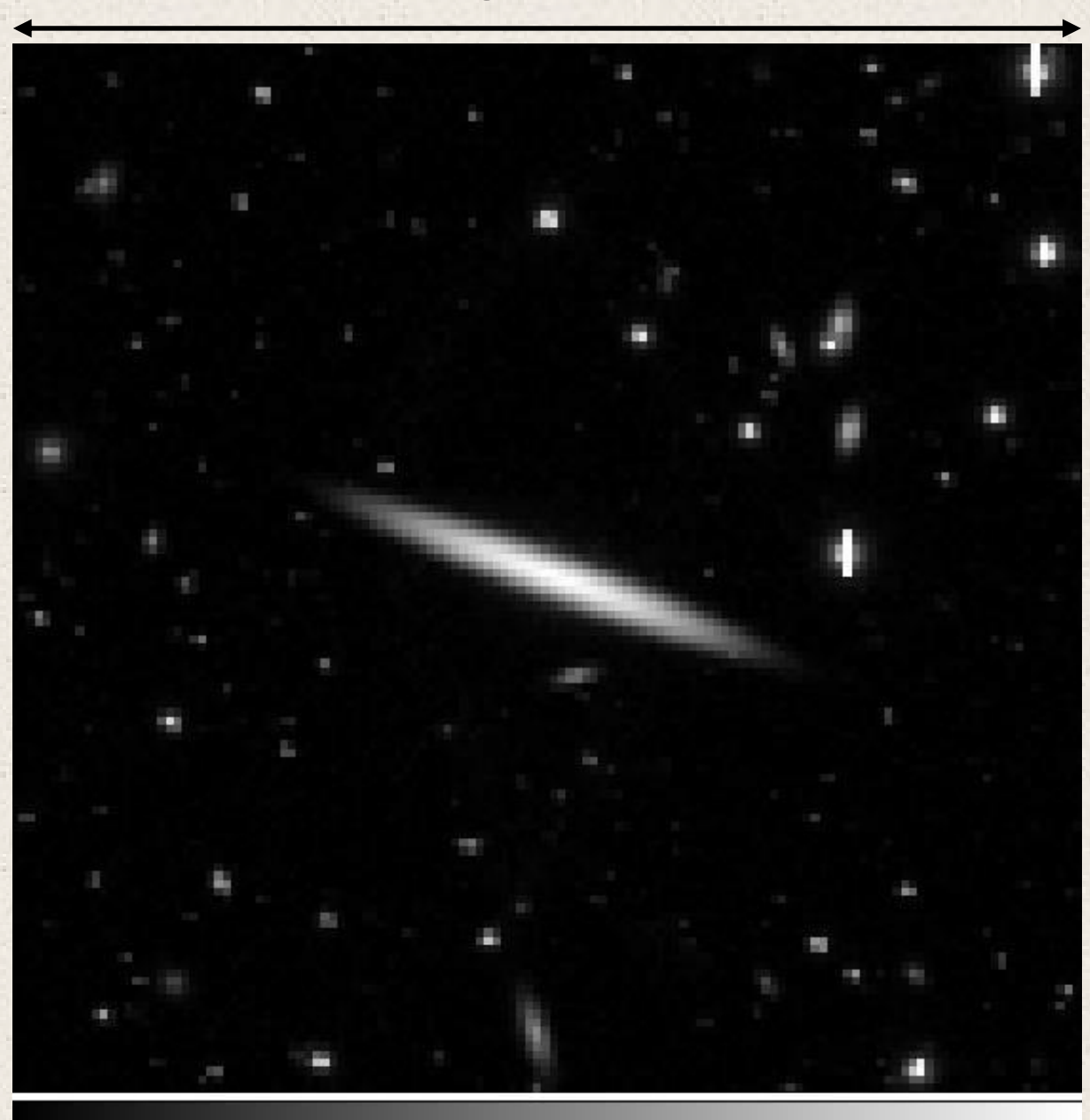

Dimension of field: $\quad 5^{\prime} \times 5^{\prime}$ Brightness of galaxy: 11 mag

Total exp time $\sim 6 \mathrm{~min}$ 


\section{U-LSB objects drift scan simulation $\sim 5$}
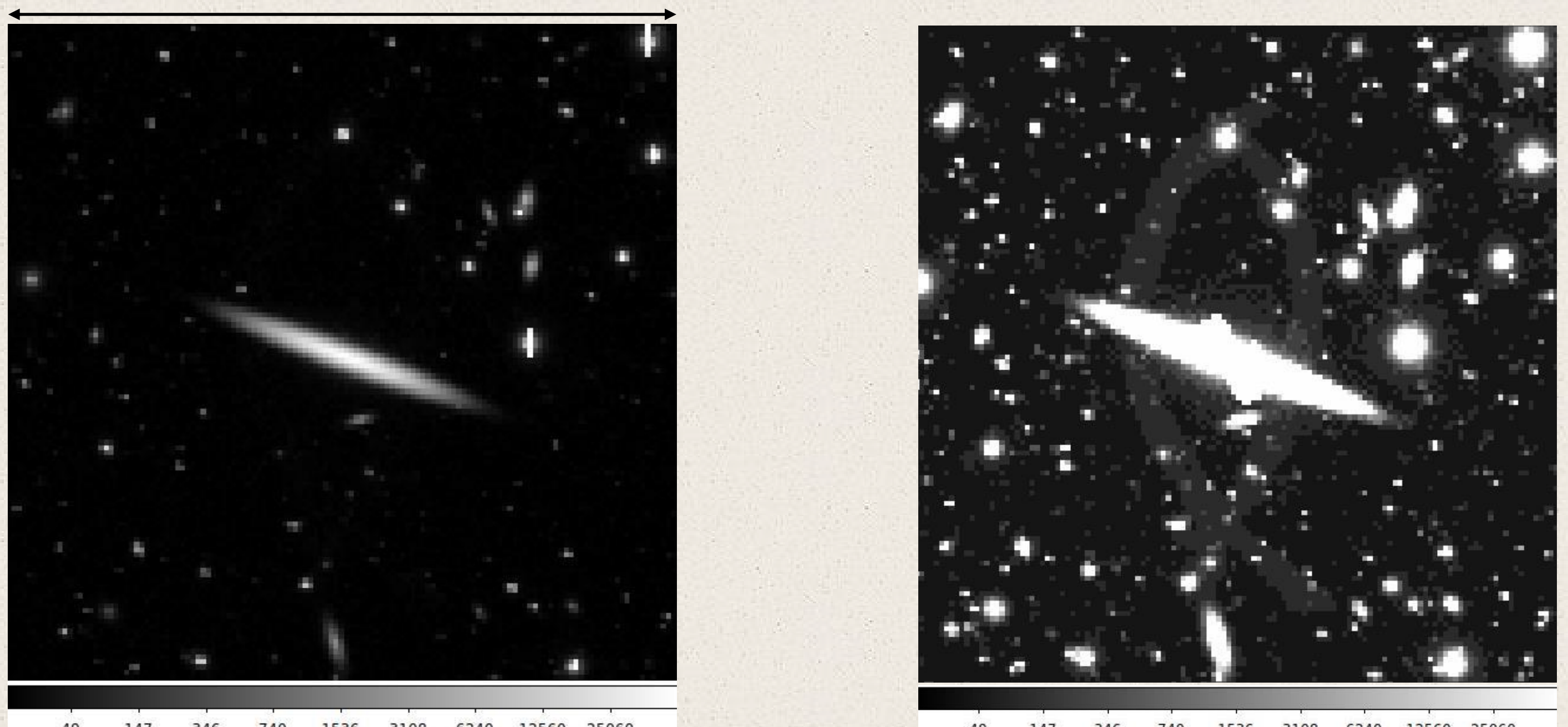

$\begin{array}{lllllllll}49 & 147 & 346 & 740 & 1536 & 3108 & 6240 & 12560 & 25060\end{array}$

Total exp time $\sim 6 \min$

Total exp time $\sim 6 \mathrm{hr}$ 


\section{U-LSB objects drift scan simulation}

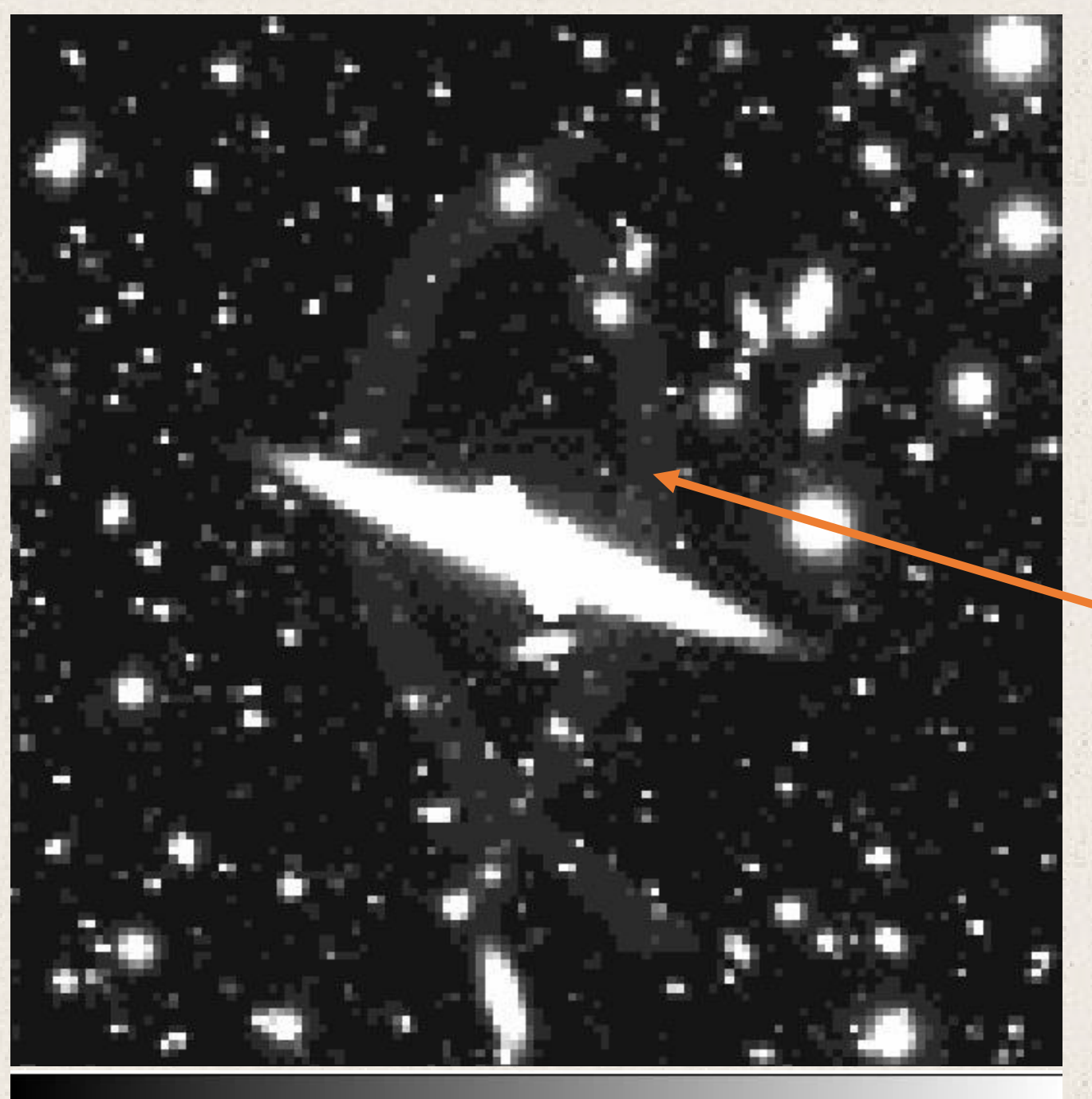

Total exp time:

$\sim 6 \mathrm{hr}$

Dimension of field: $\quad 5^{\prime} \times 5^{\prime}$

Brightness of arches: 29 mag/arcsec ${ }^{2}$ 


\section{Next steps}

- Collaboration with CEA/Leti to prototype curved CMOS

- Founding obtained by MERAC foundation (curved CCD)

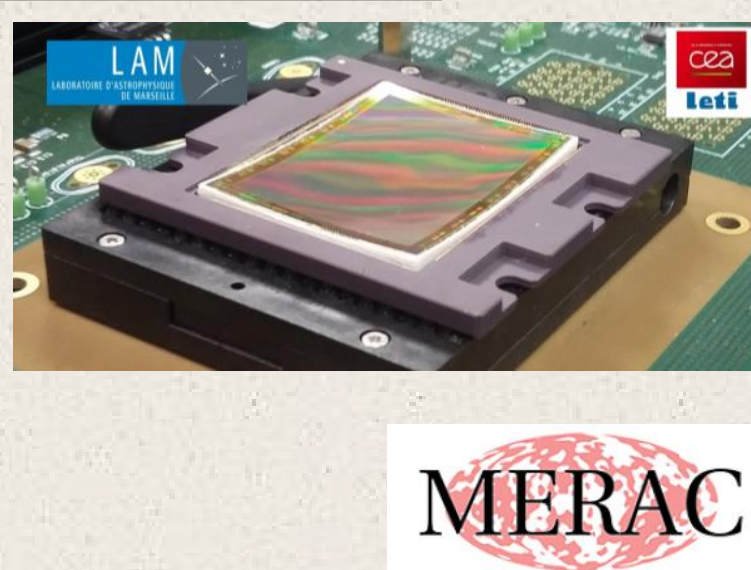

- Start prototype activity January 2019 (discussion with Teledyne E2V) (

- First prototype spring 2019

- Build and test the system in the lab, commissioning and observe! 